\title{
Evaluación interdimensional de impactos ambientales sobre la dimensión física ocasionados por cultivos de palma aceitera y la ganadería extensiva en la selva húmeda tropical del Bajo Atrato, Chocó, Colombia
}

\author{
Interdimensional evaluation of environmental impacts on the physical dimension \\ caused by cultures of oil palm and the extensive cattle in the tropical rain forest \\ of the Bajo Atrato, Chocó, Colombia
}

\author{
Teófllo Cuesta-B orja ${ }^{1}$, Glovanny Ramirez-Moreno²
}

\begin{abstract}
RESUMEN
Se presenta un análisis interdimensional de daños ambientales ocasionados por proyectos palmeros y ganaderos en el Bajo Atrato. El proyecto se desarrolló en dos fases; la primera consolidó la líne a base, en la segunda, se evaluaron los impactos ambientales mediante tres subfases: identificación de impactos, descripción de impactos por medio del modelo analítico por dimensiones y valoración y calificación de los impactos. Los resultados del proyecto indican reducción de la disponibilidad del agua en el ámbito local, alteración de la red de drenaje natural de los suelos y erosión del suelo en riberas del río Curvaradó. Se concluye que los proyectos palmeros y ganaderos son incompatibles con las condiciones agroecológicas de la zona de estudio lo que se sustenta en el hecho de que tanto el monocultivo de la palma aceitera como las pasturas, son muy exigentes en cuanto a suelos óptimos, los cuales son casi inexistentes en la zona por sus condiciones ambientales, por el alto contenido de humedad, a menos que se haga una rigurosa intervención en el medio para lograr los requerimientos de la palma y las pasturas y ello implica enormes impactos sobre el medio natural y el humano.
\end{abstract}

Palabras clave: Impacto ambiental; Dimensión física; Bajo Atrato; Modelo analítico por dimensiones; Agua; Suelo.

\begin{abstract}
This article presents an interdimensional analysis of environmental damages caused by oil palms projects and cattle in the Low Atrato. The project was developed in two phases; in first a line of base consolidated, whereas in second, the environmental impacts were evaluated; by three sub-phases: Identification of impacts, description of impacts by the analytical model by dimensions and finally, occurred the valuation and qualification of the impacts. The results of the project indicate: reduction of the water availability in the local scope; alteration of the network of natural drainage of soil and erosion of the soil in the shores of the Curvarado River. As conclusion, the oil palm and cattle projects are incompatible with the agro-ecological conditions of the zone of study; this conclusion is sustained in the fact that as much the monoculture of the oil palm as the pastures are very demanding as far as optimal soil, which does not exist for the conditions of the zone, due to its high moisture content, unless a rigorous intervention in means becomes to obtain the requirements of
\end{abstract}

1. Ingeniero Agrónomo, Especialista en Gestión Ambiental; Universidad Tecnológica del Chocó. Presidente Fundación Agricultura con Enfoque Humano (FAEH), Quibdó, Colombia.

e-mail: tcuesta@earth.ac.cr tcuesta@iiap.org.co

2. Biólogo, Magíster en Ciencias Biológicas (C). Investigador principal Componente Ecosistémico, Instituto de Investigaciones Ambientales del Pacífico (IIAP), Quibdó, Colombia. e-mail: gramirezm3@hotmail.com Recibido: enero 28, 2009

Aceptado: febrero 11, 2009 


\section{Bioetnia Volumen 6 № 1 (enero-junio), 2009}

the palm and the pastures and it implies enormous impacts on natural means and the human.

Keywords: Environmental impact; Physical dimension; Bajo Atrato; Analytical model by dimensions; Water; Soil.

\section{INTRODUCCIÓN}

La zona del Bajo Atrato por sus condiciones biofísicas, alberga ecosistemas y especies que las convierten en una de las zonas más ricas en biodiversidad de Colombia, una importante gama de ecosistemas terrestres y acuáticos configurados por un gran mosaico de comunidades y especies de flora y fauna particularmente importantes, no sólo desde el punto de vista sociocultural, sino también desde la perspectiva de su función ecológica en este complejo biológico que constituye el área de estudio. No obstante este panorama ambiental, desde hace algunos años la zona de estudio se convirtió en escenario de múltiples conflictos ambientales, entendido lo ambiental desde una perspectiva compleja que incluye el medio natural y el humano, provocando con ello claras violaciones a los derechos humanos, en particular a las comunidades afrodescendientes de estos territorios colectivos. De acuerdo con diversos estudios realizados, allí se presentaron impactos como el cambio del paisaje del bosque húmedo tropical propio de la cuenca del Atrato por un paisaje homogéneo de plantaciones de palma africana, con las configuraciones en el suelo, cauces y estructura social que dicho desarrollo implica, afectando negativamente las condiciones productivas, ecológicas necesarias para la gobernabilidad y el bienestar de las poblaciones locales. En virtud de la ocurrencia de estos hechos se presentan múltiples denuncias por parte de algunas organizaciones de la sociedad civil, de las mismas comunidades afectadas e instituciones públicas de control, lo cual dio origen a la Resolución Defensorial 039/ 2005, donde se presenta la investigación preliminar sobre las consecuencias de estas intervenciones de actores externos al territorio por la expansión de cultivos de palma aceitera en la zona, así como las responsabilidades de la institucionalidad pública sobre el particular, en aras de iniciar un proceso de reparación, compensación y protección de los derechos de las comunidades negras afectadas por estos proyectos productivos en la zona de estudio.

En consecuencia, y partiendo de la premisa de que cualquier proyecto productivo que haga uso de los recursos naturales genera impactos positivos y/o negativos, se cree que los proyectos productivos relacionados con el cultivo de la palma aceitera y la ganadería extensiva que se desarrollan en el área de estudio no son la excepción; sin embargo, y muy a pesar de todo lo que se ha dicho y escrito sobre el tema, no se conoce con certeza la verdadera situación ambiental en el área de influencia de dichos proyectos. En este contexto, la realización de una evaluación ambiental que permita establecer la verdadera dimensión de los daños ambientales ocasionados por dichos proyectos productivos, constituye una herramienta de gran utilidad para la toma de decisiones. En este sentido, se llevó a cabo una evaluación interdimensional de los daños ambientales ocasionados por los proyectos de palma aceitera y ganadería en el Bajo Atrato y en esta ocasión se presentan los resultados sobre la dimensión física.

\section{ÁREA DE ESTUDIO}

El área de estudio de la investigación se circunscribe a las comunidades negras e indígenas de los municipios Carmen de Darién y Riosucio (Chocó) y Mutatá (Antioquia) (Figura 1).

\section{MÉTODOS}

El proyecto se desarrolló en dos fases, la primera tuvo que ver con la consolidación de la línea base para la dimensión física y la segunda se refiere a la evaluación de los impactos ambientales, en tres subfases, identificación, descripción y valoración de los impactos. A continuación, se detallan cada una de estas fases y subfases:

Consolidación de la línea base. La línea base sobre la dimensión física incluye información sobre el recurso agua, suelo y aire; sin embargo, para el caso de este estudio, sólo se tomaron en cuenta los dos primeros aspectos (agua y suelo), porque fueron los afectados en mayor medida por los proyectos productivos de palma aceitera y ganadería extensiva.

Valoración de impactos ambientales. Esta fase está constituida por tres subfases, a saber: identificación, descripción y valoración de impactos. A continuación, se describen cada:

Identificación de impactos ambientales. La identificación de los impactos ambientales partió de un análisis comparativo entre los indicadores cuantitativos y cualitativos de las diferentes variables ambientales, tomando como base la información de línea base y el diagnóstico ambiental. Para el logro del propósito, se aplicaron varias herramientas metodológicas, entre las cuales se destacan ejercicios, cartografía social participativa y visitas técnicas de campo por parte de un equipo interdisciplinario vinculado con el proyecto.

Descripción de impactos ambientales. La descripción de los impactos ambientales consistió en ponerle atributos cualitativos a los impactos, al tiempo que se realizó un análisis de los mismos desde una perspectiva integral. En este ejercicio se aplicó la herramienta metodológica planteada por el modelo analítico por dimensiones, la cual cosiste en un cruce de la física con las dimensiones biótica, económica, cultural y 


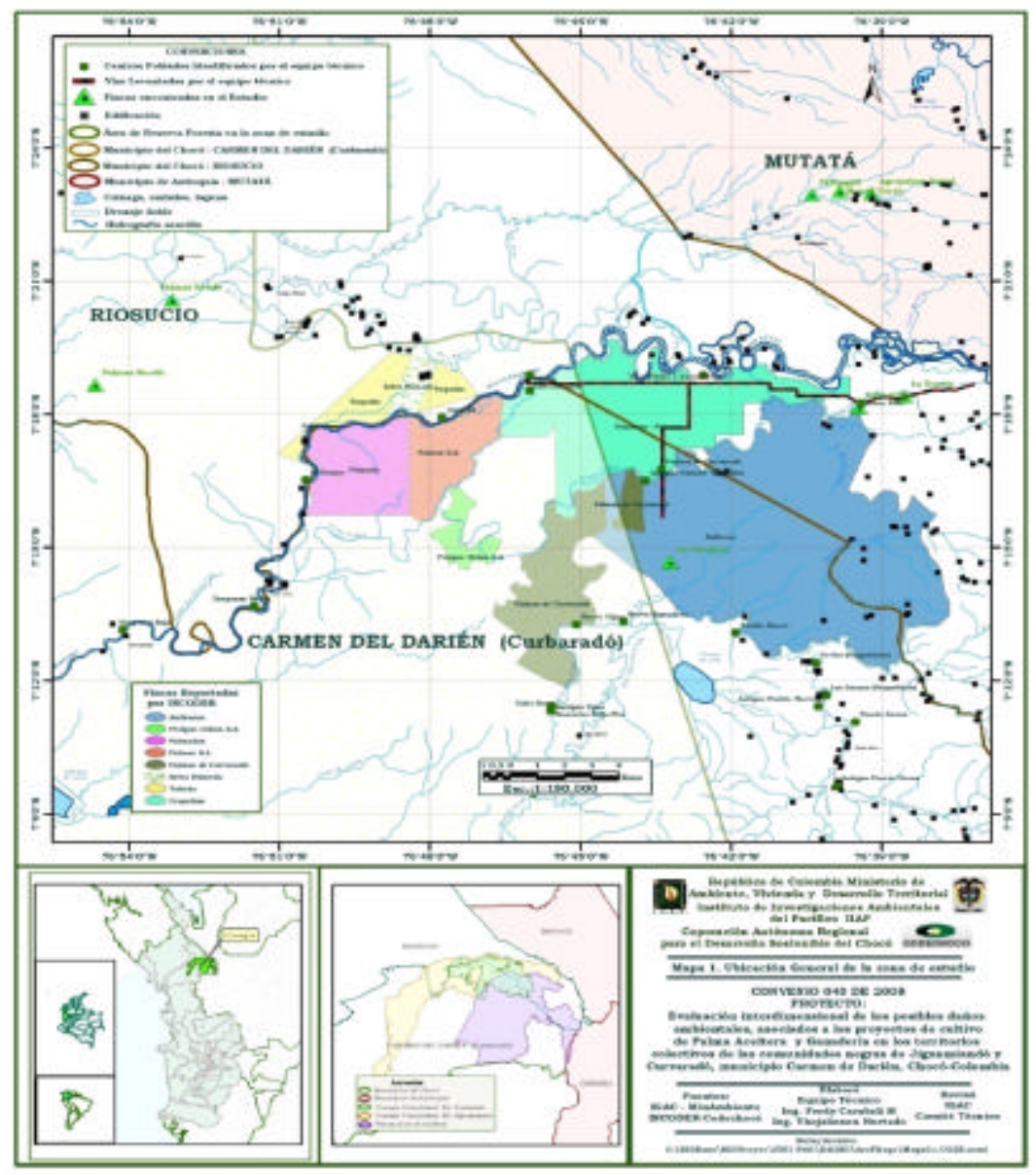

Figura 1. Ubicación general del área de estudio

política, tal como se describe en la Tabla 1.

Valoración de impactos ambientales. La valoración de los impactos ambientales sobre la dimensión física se dio para las diferentes variables ambientales impactadas, no sólo desde la perspectiva cualitativa, sino también desde el punto de vista cuantitativo. Para ello, se aplicaron varias herramientas metodológicas, entre las cuales se encuentran el análisis químico de aguas en el laboratorio de CORPOURABA, con el fin de establecer posibles niveles de contaminación de las aguas en las fincas palmeras y ganaderas. De otro lado, es importante anotar que la valoración cuantitativa de los impactos ambientales se complementó con una calificación de los mismos en térmicos de impactos bajos, medios, altos y muy altos, dependiendo el caso, y con base en el conocimiento del equipo técnico del proyecto.

\section{RESULTADOS Y DISCUSIÓN}

La dimensión física recibió varios impactos ambientales a raíz de algunas acciones de intervención llevadas a cabo por las empresas palmeras y ganaderas en la zona de estudio. A continuación se presentan los impactos identificados y analizados para la dimensión física:

Impactos ambientales sobre la dimensión física.Entre los impactos ambientales sobre esta dimensión se destacan la reducción de la disponibilidad de agua para comunidades locales, erosión del suelo en las riberas del río Curvaradó, reducción en el nivel freático de los suelos intervenidos y afectación de la red de drenaje natural de los suelos intervenidos, tal como se detalla a continuación:

Reducción de la disponibilidad del agua en el ámbito local. La disponibilidad hídrica local se refiere a la cantidad de agua a la cual puede acceder fácilmente una determinada población de personas; dicho impacto se ve reflejado en un menor acceso al agua por parte de las comunidades establecidas en el área de estudio. Este impacto, tiene su origen en una serie de acciones de intervención en la zona de estudio, entre las cuales se encuentran el taponamiento, drenaje y desvío de cuerpos de agua; construcción de una amplia red de drenajes artificiales y la eliminación de cobertura boscosa (Tabla 2). El mencionado impacto generó una serie de efectos tanto para la misma dimensión física, como para las otras 
Bioetnia Volumen 6 № 1 (enero-junio), 2009

Tabla 1

Análisis interdimensional desde la dimensión física

\begin{tabular}{llllll}
\hline Dimensiones & \multicolumn{1}{c}{ Física } & \multicolumn{1}{c}{ Biótica } & Cultural & Económica & Política \\
\hline Física & Procesos y & Adaptación de & Adaptación & Posibilidades y & Importancia \\
& rasgos físicos & comunidades & humana / & restricciones / & estratégica / \\
& del medio & bióticas / & diversidad y & valor de uso & conflictos de \\
& natural & especiación y & territorio & & uso y territorio \\
& & & & \\
\hline
\end{tabular}

Fuente: Ángel et al. 2007

Tabla 2

Valoración y calificación de impactos ambientales ocasionados por las empresas palmeras y ganaderas

\begin{tabular}{|c|c|c|c|}
\hline \multirow[t]{3}{*}{ Empresas } & \multicolumn{3}{|c|}{ I mpacto sobre reducción en la disponibilidad de agua en el ámbito local } \\
\hline & \multirow{2}{*}{$\begin{array}{l}\text { Valoración cuantitativa } \\
\text { № de cuerpos de agua } \\
\text { afectados }\end{array}$} & \multicolumn{2}{|c|}{ Valoración cualitativa } \\
\hline & & Descripción del impacto & $\begin{array}{l}\text { Calificación } \\
\text { del impacto* }\end{array}$ \\
\hline URAPALMA S.A. & 10 & $\begin{array}{l}\text { La empresa URAPALMA S.A. afectó el } \\
\text { caudal de } 10 \text { cuerpos de agua, entre los } \\
\text { cuales se encuentran Caño el Cerrao, } \\
\text { Quebrada la Morroca, Quebrada la Pradera, } \\
\text { Quebrada la Nevera, Quebrada la I guana, } \\
\text { Quebrada la Madre, Quebrada la Cristalina, } \\
\text { Ciénaga Alvarado, Ciénaga los Cativos y } \\
\text { Ciénaga la Cristalina. }\end{array}$ & Muy alto \\
\hline Palmas de Curvaradó & 1 & $\begin{array}{l}\text { La empresa Palmas de Curvaradó ocasionó } \\
\text { el secado irreversible de la ciénaga Andalu- } \\
\text { cía. }\end{array}$ & Muy alto \\
\hline Palmados & 1 & $\begin{array}{l}\text { La empresa Palmados ocasionó el secado } \\
\text { irreversible de la ciénaga el Guacuco. }\end{array}$ & Muy alto \\
\hline Palmas S.A. & 1 & $\begin{array}{l}\text { La empresa Palmas S.A. ocasionó el secado } \\
\text { irreversible de la ciénaga los Bartolos. }\end{array}$ & Muy alto \\
\hline Tukeka & 1 & $\begin{array}{l}\text { La empresa ganadera Tukeka canalizó el } \\
\text { caño el Cojo, reduciendo su caudal. }\end{array}$ & Bajo \\
\hline Selva Húmeda & 0 & Empresa ganadera proyectada & Nulo \\
\hline Asibiscon & 0 & Empresa palmera proyectada & Nulo \\
\hline Inversiones Freigne Ochoa & 0 & Empresa palmera proyectada & Nulo \\
\hline
\end{tabular}

*Afectación de 1-2 cuerpos de agua sin secado irreversible de alguno de estos (Bajo); afectación de 2-3 cuerpos de agua sin secado irreversible de alguno de estos (Medio); afectación 3-5 cuerpos de agua sin secado irreversible de alguno de estos (Alto); Afectación de más de 5 cuerpos de agua ó secado irreversible de algún cuerpo de agua (Muy Alto) 


\section{Evaluación interdimensional de impactos ambientales. T. Cuesta-Borja, G. Ramírez-Moreno}

dimensiones, es decir biótica, económica, cultural y política.

Efectos sobre la dimensión física. Reducción del caudal de 12 cuerpos de agua, entre los cuales se encuentran la quebrada Cristalina, ciénaga la Cristalina, caño el Cerrao, caño Claro, quebrada la Nevera, quebrada la Pradera, quebrada la Madre, quebrada la Morroca, quebrada la Iguana, caño el Cojo, ciénaga el Cojo, y la ciénaga el Guacuco y el secado irreversible de 4 ciénagas, entre las que se encuentranAndalucía, Alvarado, los Bartolos y los Cativos.

Efectos sobre la dimensión biótica. A partir de la reducción del caudal o en su defecto el secado irreversible de los 16 cuerpos de agua, se registra una serie de efectos sobre la dimensión biótica, entre los cuales sobresalen la destrucción de hábitats naturales de especies de flora y fauna, emigración de especies de flora y fauna, ruptura de cadenas tróficas, entre otros efectos.

Efectos sobre la dimensión económica. Los efectos sobre la dimensión económica están representados básicamente en la afectación de la actividad económica de la pesca artesanal, a través de la cual la mayoría de la población local derivaba parte de sus ingresos por concepto de la venta de pescados; adicionalmente, se afectó la seguridad alimentaria, porque la pesca artesanal reducía los costos de alimentación de las familias locales. Por último, generó un efecto en la población que hacía uso de al menos 3 de los 16 cuerpos de agua afectados para el transporte acuático en pequeñas embarcaciones; sin embargo, con el secado de estos, no sólo se incrementan los tiempos y costos de transporte entre algunas poblaciones, sino que también se afectó el intercambio comercial entre las comunidades.

Efectos sobre la dimensión cultural. En esta dimensión se presentó una serie de efectos, entre los cuales se encuentran la afectación de prácticas tradicionales como la pesca artesanal, el transporte en canoas y la medicina tradicional, esta última práctica se ve afectada en virtud de que los cuerpos de agua permitían el traslado de enfermos de manera ágil para recibir tratamientos basados en la medicina tradicional; también, se afectó la conectividad entre las comunidades, lo cual facilitaba el intercambio cultural entre estas.

Efectos sobre la dimensión política. El impacto ambiental en consideración tuvo varios efectos sobre la dimensión política; sin embargo, los efectos más sobresalientes se dieron en términos legales, es decir, se violó la normatividad ambiental colombiana, como por ejemplo el Artículo 86 del Decreto Ley 2811/74, el cual prohíbe el taponamiento, desvío y drenaje de cuerpos de agua naturales. También se violó la Ley 70/93 y el Decreto 1745/95, los cuales otorgan la administración de territorios a las comunidades negras; sin embargo, no se consultó con dichas comunidades la intervención en su propio territorio.

Afectación de 1-2 cuerpos de agua sin secado irreversible de alguno de estos (Bajo); afectación de 2-3 cuerpos de agua sin secado irreversible de alguno de estos (Medio); afectación 3-5 cuerpos de agua sin secado irreversible de alguno de estos (Alto); Afectación de más de 5 cuerpos de agua ó secado irreversible de algún cuerpo de agua (Muy alto)

Finalmente, es importante anotar que de la empresa palmera URAPALMA se derivaron las empresas Palmura e Inversiones Agropalma; sin embargo, estas fueron establecidas por URAPALMA, por tanto los impactos generados por las acciones en la fase de establecimiento de los cultivos de palma son atribuibles a la empresa matriz.

Alteración de la red de drenaje natural del suelo. Este impacto ambiental se define como la modificación en el flujo natural de las aguas en el suelo, las cuales fluyen en función de variables como la topografía y composición física de los suelos. En este sentido, se considera que dicho impacto ambiental tiene su origen en la construcción de una amplia red de drenajes artificiales por las empresas palmeras y ganaderas en la zona de estudio; dicho flujo fue alterado, con lo cual se afectó la disponibilidad de agua en algunas fuentes hídricas que recibían el agua subterránea (Tabla 3). El anterior impacto ambiental, generó varios efectos sobre las dimensiones física, biótica, económica, cultural y política, tal como se detalla a continuación.

Efectos sobre la dimensiónfísica. La interrupción del flujo natural de las aguas en los suelos intervenidos, en algunos casos reduce el caudal de los cuerpos de agua que recibían las aguas subterráneas, mientras que otros cuerpos de agua incrementan su caudal, en virtud de los mayores aportes provenientes de los acuíferos subterráneos.

Efectos sobre la dimensión biótica. Con la reducción del caudal hídrico de algunos cuerpos de agua se desfavorece la permanencia de algunas especies fáunicas que requieren ciertos niveles de agua para llevar a cabo sus procesos vitales y reproductivos; por su parte, con el incremento del caudal de otros cuerpos de agua se favorece la llegada de algunas especies animales que requieren suficiente cantidad de agua para cumplir con sus funciones vitales y reproductivas. Adicionalmente, con la disminución o incremento de los niveles de humedad en ciertas franjas de suelo, en virtud de la alteración del drenaje natural de las aguas, se afecta la permanencia de algunas especies de flora y fauna que requieren de ciertos niveles de humedad, con lo cual se podría presentar desequilibrios ecológicos, al tiempo que se perjudicaría los niveles de biodiversidad del suelo en el ámbito local.

Efectos sobre la dimensión económica. Con la interrupción del flujo natural de las aguas del suelo y la consiguiente afectación del caudal de algunos cuerpos de agua que albergan altos niveles poblacionales de peces, se podría ver disminuida la actividad económica de la pesca artesanal, con lo cual no sólo se reducirían los ingresos familiares, sino que también se afecta la seguridad alimentaria de los pobladores locales. 


\section{Tabla 3 \\ Afectación del flujo natural de las aguas del suelo por parte de las empresas palmeras y ganaderas de acuerdo con el tamaño de la red de drenajes construidos}

\begin{tabular}{lcc}
\hline \multicolumn{1}{c}{ Empresas } & \multicolumn{2}{c}{ I mpacto sobre alteración del drenaje natural de los suelos } \\
\cline { 2 - 3 } & $\begin{array}{c}\text { Valoración cuantitativa } \\
\text { km de drenajes construidos }\end{array}$ & Calificación del impacto* \\
\hline URAPALMA S.A. & 738,19 & Muy alto \\
\hline Palmas de Curvaradó & 382,1 & Muy alto \\
\hline Palmados & 270,2 & Muy alto \\
\hline Palmas SA & 205,7 & Muy alto \\
\hline Tukeka & ND & - \\
\hline Selva húmeda & ND & - \\
\hline Asibiscon & ND & - \\
\hline Inversiones Freigne Ochoa & 69,9 & Medio \\
\hline
\end{tabular}

0-50 km (bajo); 50-100 km (medio); 100-150 km (alto) Más de 150 km (muy alto) ND. Información no disponible

Efectos sobre la dimensión cultural.Con la reducción del caudal del ciertos cuerpos de agua, en virtud de la alteración del flujo natural de las aguas del suelo se podrían ver afectadas varias prácticas culturales como la pesca artesanal, el transporte acuático por algunos cuerpos de agua que en condiciones normales eran navegables y por ende se afectaría el intercambio cultural entre algunas comunidades. Adicionalmente, la reducción o incremento de los niveles de humedad de los suelos de la zona podría favorecer o desfavorecer la actividad agrícola tradicional con todas las implicaciones sociales asociadas con dicho efecto.

Efectos sobre la dimensión política. Con la afectación del drenaje natural de las aguas del suelo y la consiguiente reducción o incremento de los caudales en ciertos cuerpos de agua de la zona de estudio, se podría estar violando la normatividad ambiental colombiana, específicamente el Artículo 86 del Decreto Ley 2811/74, en tanto que se estaría afectando la condición natural de estos cuerpos de agua.

Erosión hídrica del suelo en las riberas del Curvaradó. La erosión hídrica del suelo se define como el proceso mediante el cual se da la pérdida del suelo por acción de las aguas, en este caso del río Curvaradó, debido al incremento del caudal del río y desprotección de las riberas. Este impacto ambiental se generóa raíz de una serie de actividades de intervención llevadas a cabo en la zona de estudio, entre las cuales se destacan el taponamiento del caño el Cerrao, eliminación de cobertura vegetal en las riberas del Curvaradó y la extracción de material de arrastre en el mismo río (Tabla 4). El mencionado impacto ambiental, generó una serie de efectos directose indirectos sobre las dimensiones física, biótica, económica, cultural y política, tal como se detalla a continuación.
Efectos sobre la dimensión física. Con la erosión del suelo en las riberas del Curvaradó se dio un notable incremento en los niveles de sedimentación en las aguas de este río, lo cual no sólo sugiere altos niveles de contaminación físico-química de las aguas, sino que también favorece procesos de inundaciones en la zona por efecto de la alteración del área de conducción de las aguas; adicionalmente, el fenómeno de la erosión hídrica del suelo, no sólo contribuye a la degradación de dicho recurso debido a la pérdida de la capa orgánica, sino que también se pierden grandes áreas, para ilustrar el fenómeno de la erosión del suelo en las riberas del Curvaradó.

Efectos sobre la dimensión biótica. Con el incremento de los niveles de sedimentación en las aguas del Curvaradó se infiere que hay una afectación directa del hábitat de varios grupos fáunicos como peces y reptiles, los cuales se encuentran asociados con dicho ecosistema. De igual manera, con la erosión del suelo se afecta su biología en dos sentidos; en primer lugar, con la pérdida del horizonte orgánico del suelo se perjudica la permanencia del grupo de insectos del orden coleóptero, los cuales se alimentan de la materia orgánica en descomposición; en segunda medida, se da una pérdida de especies de flora y fauna que se encuentran en el área erosionada.

Efectos sobre la dimensión económica. Como producto de la presencia de sedimentos en el agua, los pobladores están incurriendo en mayores costos para el acceso al agua, no sólo porque han tenido que dotarse de los implementos necesarios para el almacenamiento de las aguas lluvias, sino también para el tratamiento artesanal del agua del río en época de verano, es decir en ausencia de aguas lluvias. De otro lado, es importante anotar que con la sedimentación del río y el 
Tabla 4

Valoración y calificación del impacto ambiental sobre erosión hídrica de los suelos en las riberas del Curvaradó

\begin{tabular}{|c|c|c|c|}
\hline \multirow[t]{3}{*}{ Empresas } & \multicolumn{3}{|c|}{ Impacto sobre erosión hídrica de los suelos en las riberas del Curvaradó } \\
\hline & \multirow{2}{*}{$\begin{array}{l}\text { Valoración cuantitativa } \\
\text { № de acciones que } \\
\text { ocasionan erosión }\end{array}$} & \multicolumn{2}{|c|}{ Valoración cualitativa } \\
\hline & & Descripción del impacto & $\begin{array}{l}\text { Calificación } \\
\text { del impacto* }\end{array}$ \\
\hline URAPALMA SA & 3 & $\begin{array}{l}\text { Extracción de material de playa del río } \\
\text { Curvaradó, deforestación y taponamiento } \\
\text { caño el Cerrao }\end{array}$ & Alto \\
\hline Palmas de Curvaradó & 1 & $\begin{array}{l}\text { Extracción de material de playa del río } \\
\text { Curvaradó }\end{array}$ & Bajo \\
\hline Palmados & 2 & $\begin{array}{l}\text { Extracción de material de playa del río } \\
\text { Curvaradó y deforestación }\end{array}$ & Medio \\
\hline Palmas SA & 2 & $\begin{array}{l}\text { Extracción de material de playa del río } \\
\text { Curvaradó y deforestación }\end{array}$ & Medio \\
\hline Tukeka & 1 & Deforestación & Bajo \\
\hline Selva húmeda & ND & No se reporta & - \\
\hline Asibiscon & ND & No se reporta & - \\
\hline $\begin{array}{l}\text { Inversiones Freigne } \\
\text { Ochoa }\end{array}$ & 1 & Deforestación & Bajo \\
\hline
\end{tabular}

1 acción (bajo); 2 acciones (medio); 3 acciones (alto)

consiguiente incremento de las inundaciones de las tierras agrícolas, no sólo se ve disminuida dicha actividad económica, sino que también afecta la seguridad alimentaria local.

Efectos sobre la dimensión cultural. Con los altos niveles de sedimentación del río Curvaradó se aumenta la frecuencia de inundación de las tierras ribereñas, las cuales son utilizadas tradicionalmente para la práctica de la agricultura familiar; sin embargo, con este fenómeno se hace imposible ejercer la agricultura tradicional, afectando con ello una tradición de gran contenido simbólico en la cultura afrochocoana. También con el fenómeno de la erosión del suelo se generó un efecto directo en algunos poblados establecidos en las riberas del Curvaradó, porque algunas viviendas hoy se encuentran en alto riesgo, por la amenaza permanente de que el río las arrastre. Ello, podría tener un efecto de largo plazo en la permanencia de algunos poblados, entre los cuales se encuentran el Guamo, Despensa Media y Despensa Baja.

Efectos sobre la dimensión política. En cuanto a los efectos sobre esta dimensión se puede registrar el hecho de que los altos niveles de sedimentación de las aguas del Curvaradó, podrían sobrepasar los niveles máximos permitidos por la legislación ambiental colombiana.

Reducción del nivel freático de los suelos intervenidos. El impacto ambiental sobre afectación del nivel freático de los suelos consiste en el descenso del nivel de las aguas subterráneas del suelo, debido a varias acciones de intervención, entre las cuales se encuentran la construcción de una amplia red de drenajes artificiales, canalización, taponamiento y desvío de cuerpos de agua y la eliminación de cobertura boscosa por parte de las empresas palmeras y ganaderas en la zona de estudio (Tabla 5). Dicho impacto ambiental generó varios efectos sobre las dimensiones física, biótica, económica, cultural y política.

Efectos sobre la dimensión física. Con la disminución del nivel freático de los suelos intervenidos en al menos 100 centímetros se afectó no sólo la disponibilidad de agua en dichos suelos, sino también la presencia de algunos elementos químicos que permanecían disueltos en las aguas subterráneas al alcance de los cultivos; sin embargo, con la reducción del nivel freático se incrementan las pérdidas de nutrientes químicos por efecto de una mayor percolación de las aguas lluvias, porque el nuevo nivel freático o tabla de agua ahora permanece por debajo del alcance de las rices de los cultivos.

Efectos sobre la dimensión biótica. La disminución del nivel freático de los suelos en la zona de estudio, generó una serie de efectos biológicos, entre los cuales se registran la pérdida de hábitat natural de algunas especies de flora como por ejemplo el Chacarrá (Bactris brongniartii), Naidi(Euterpe 
Tabla 5

Valoración y calificación del impacto ambiental sobre reducción del nivel freático de los suelos

\begin{tabular}{|c|c|c|c|}
\hline \multirow[t]{3}{*}{ Empresas } & \multicolumn{3}{|c|}{ Impacto sobre reducción del nivel freático de los suelos } \\
\hline & \multirow{2}{*}{$\begin{array}{l}\text { Valoración cuantitativa } \\
\text { № de acciones asociadas } \\
\text { con el impacto }\end{array}$} & \multicolumn{2}{|c|}{ Valoración cualitativa } \\
\hline & & $\begin{array}{l}\text { Descripción de acciones } \\
\text { involucradas }\end{array}$ & $\begin{array}{l}\text { Calificación } \\
\text { del impacto* }\end{array}$ \\
\hline URAPALMA SA & 5 & $\begin{array}{l}\text { Construcción de drenajes artificiales, } \\
\text { taponamiento de caños y quebradas, } \\
\text { canalización de caños y quebradas, } \\
\text { desvío de caños y quebradas y } \\
\text { deforestación }\end{array}$ & Alto \\
\hline Palmas de Curvaradó & 2 & $\begin{array}{l}\text { Canalización de la ciénaga Andalucía, } \\
\text { construcción de drenajes artificiales }\end{array}$ & Medio \\
\hline Palmados & 3 & $\begin{array}{l}\text { Deforestación, construcción de drenajes } \\
\text { artificiales, canalización de la ciénaga el } \\
\text { Guacuco }\end{array}$ & Medio \\
\hline Palmas SA & 3 & $\begin{array}{l}\text { Construcción de drenajes artificiales, } \\
\text { deforestación, canalización de ciénaga los } \\
\text { Bartolos }\end{array}$ & Medio \\
\hline Tukeka & 3 & $\begin{array}{l}\text { Construcción de drenajes artificiales, } \\
\text { canalización Caño el Cojo, deforestación }\end{array}$ & Medio \\
\hline Selva Húmeda & ND & - & - \\
\hline Asibiscon & 1 & Deforestación & Bajo \\
\hline $\begin{array}{l}\text { Inversiones Freigne } \\
\text { Ochoa }\end{array}$ & 2 & $\begin{array}{l}\text { Deforestación, construcción de drenajes } \\
\text { artificiales }\end{array}$ & Medio \\
\hline
\end{tabular}

1 (bajo); 2-3 acciones (medio); 4-5 acciones (alto); más de 5 acciones (muy alto) ND no disponible

predatoria) y Palmilla (Geonoma triandra), todas pertenecientes a la familia Arecaceae y otras especies de la familia Araceae como Anthurium formosum, Anthurium Bakeri, Monstera sp., Philodendronfragantisimum y Philodendron fragantisimum, las cuales requieren de terrenos con un nivel freático alto para su normal desarrollo y sostenibilidad en el tiempo. Adicionalmente, se afectó el hábitat de algunas especies de fauna, entre las cuales se destacan los anfibios y reptiles.

Efectos sobre la dimensión económica. Con la reducción de algunas comunidades de palmas propias de ecosistemas pantanosos, se afectó la economía de quienes aprovechaban estas especies no sólo para la construcción de sus propias viviendas, sino también como materia prima para la elaboración de piezas artesanales.

Efectos sobre la dimensión cultural. Con la reducción de las comunidades de palmas y otras especies de flora y fauna, se afectaron varias prácticas culturales entre las cuales se encuentran la cacería de especies animales y la construcción de viviendas tradicionales en palafitos, gracias a los materiales extraídos del bosque.

Efectos sobre la dimensión política. Con la afectación de la condición natural de ecosistemas pantanosos como el que representaba el área de estudio, se habría incurrido en violación de la normatividad ambiental colombiana, especialmente el Decreto Ley 2811/74.

\section{CONCLUSIONES}

Se logró establecer que tanto los proyectos palmeros como ganaderos en la zona de estudio generaron los mayores impactos ambientales en su fase de establecimiento, por las grandes acciones de intervención para el acondicionamiento de los suelos (eliminación de cobertura vegetal, drenaje de suelos, etc.) y construcción de red vial (carreteras y sistema de cable vías); sin embargo, se preven impactos importantes en la fase de operación de los proyectos, sólo que aún no se logran percibir, ya sea porque no se han manifestado o en su 


\section{Evaluación interdimensional de impactos ambientales. T. Cuesta-Borja, G. Ramírez-Moreno}

defecto por la carencia de información de campo que permita auscultarlos. Adicionalmente, se concluye que tanto los proyectos palmeros como ganaderos son incompatibles con las condiciones agroecológicas de la zona de estudio. Esta conclusión se sustenta en el hecho de que tanto el monocultivo de la palma aceitera como las pasturas son muy exigentes en cuanto a suelos óptimos, lo cual no existe para los condiciones de la zona, por su alto contenido de humedad, a menos que se haga una rigurosa intervención en el medio para lograr los requerimientos de la palma y las pasturas y ello implica enormes impactos sobre el medio natural y el humano.

\section{LITERATURA CITADA}

Angel, E., Carmona, S.I., Villegas, L.C. 2007. Gestión ambiental en proyectos de desarrollo. $3^{\mathrm{a}}$ ed. Medellín: UNALMED. 235 p.

Angel, E., Villegas, L.C., Carmona S.I. 1996. Gestión ambiental en proyectos de desarrollo, una propuesta desde los proyectos energéticos. Santa $\mathrm{Fe}$ de Bogotá: Fondo FEN Colombia. 290 p.

Defensoria del Pueblo. 2005. Resolución Defensorial No 039. Violación de los derechos humanos por siembra de palma africana en territorios colectivos de Jiguamiandó y Curvaradó- Chocó. Bogotá, DC: Defensoria del Pueblo; $42 \mathrm{p}$.

Presidencia de la República. Decreto No 1745 del 12 de Octubre de 1995. «Por el cual se reglamenta el Capítulo III de la Ley 70 de 1993, se adopta el procedimiento para el reconocimiento del derecho a la propiedad colectiva de las 'Tierras de las Comunidades Negras' y se dictan otras disposiciones».

Presidencia de la República. Decreto 2811 del 18 de diciembre 1974. «Pormedio del cual se crea el Código Nacional de Recursos Naturales Renovables y de Protección al Medio Ambiente».

Congreso de la República. Ley 99 del 22 de diciembre de 1993. «Por la cual se crea el Ministerio del Medio Ambiente».

Congreso de la República. Ley 2 del 16 de diciembre de 1959. «Por medio de la cual se crean 7 zonas de reserva forestal». 